\title{
Restoration of Circadian Behavior by Anterior Hypothalamic Heterografts
}

\author{
Patricia J. Sollars,, ${ }^{1,2}$ Daniel P. Kimble, ${ }^{2}$ and Gary E. Pickard ${ }^{1,3}$ \\ ${ }^{1}$ Departments of Psychiatry and ${ }^{3}$ Neuroscience, University of Pennsylvania School of Medicine, Philadelphia, \\ Pennsylvania, and 'Institute of Neuroscience, University of Oregon, Eugene, Oregon
}

The suprachiasmatic nucleus (SCN) of the anterior hypothalamus (AH) is a circadian oscillator and an important component of the mammalian circadian system. To determine whether the SCN is the dominant circadian pacemaker responsible for generating a species-typical characteristic of circadian rhythms [i.e., period length $(\tau)]$, neural transplantation was conducted using fetal AH donors of different species and SCN-lesioned (SCNx) hosts. The circadian behavior of each of the three donor species is clearly distinguishable by its species-typical $\tau$. The extent of SCN pacemaker autonomy was assessed by noting whether the period of the restored circadian rhythm following heterograft transplantation was characteristic of the donor or the host, or whether an atypical circadian period was established. Hamsters rendered arhythmic by $\mathrm{SCN}$ ablation were implanted with AH tissue from fetal hamsters (E13E14, homograft controls) or fetal mice or rats (E15-E17). The AH homografts restored circadian activity rhythms with a $\tau$ similar to that of intact hamsters, and fetal mouse AH heterografts restored circadian rhythmicity with a $\tau$ similar to that of the donor mouse strain. However, fetal rat AH tissue implanted into SCNx hamsters renewed circadian rhythmicity with a period significantly shorter than either the species-typical $\tau$ of the rat donor or the hamster host.

In both the mouse and rat AH heterograft experiments, immunocytochemical analysis performed with speciesspecific monoclonal antibodies revealed extensive fiber outgrowth from the implant into the host hypothalamus, evident up to 7 months postimplantation. The rat implants were consistently larger, more fully vascularized and exhibited less necrosis than the implanted mouse tissue. The histological appearance of the grafts, thus, provides no explanation for the difference in efficacy of the grafts to restore species-typical behavior. However, several interpre-

\footnotetext{
Received Nov. 19, 1993; revised Aug. 22, 1994; accepted Sept. 23, 1994.

This work was supported by a grant from the Medical Research Foundation of Oregon (D.P.K.), NIH Grants GM 07257 (P.J.S.), NS 21165 (G.E.P.), and MH 47501 (G.E.P.), and a grant from the University of Pennsylvania Research Foundation (G.E.P.). We thank Suzanne Sollars for expert surgical assistance, Dr. Ilene Bernstein for a critical reading of an earlier version of this manuscript, and Dr. Nathan J. Tublitz, whose enthusiasm for this work ensured its completion.

Correspondence should be addressed to Dr. Patricia J. Sollars, Center for Sleep and Respiratory Neurobiology, Hospital of the University of Pennsylvania, 991 Maloney Building, 3600 Spruce Street, Philadelphia, PA 19104 4283

Copyright $\subset 1995$ Society for Neuroscience 0270-6474/95/152109-14\$05.00/0
}

tations are considered that are consistent with the combined behavioral results observed.

[Key words: circadian rhythms, suprachiasmatic nucleus, heterografts, homografts, neural transplantation, fiber outgrowth]

The suprachiasmatic nucleus ( $\mathrm{SCN}$ ) of the anterior hypothalamus $(\mathrm{AH})$ is a critical component of the mammalian circadian system. The initial studies of Stephan and Zucker (1972) demonstrating the disruption of circadian rhythmicity following SCN ablation in the rat have been confirmed in other rodent species and in primates (Moore and Klein, 1974; Rusak, 1977; Reppert et al., 1981; Dark et al., 1985; Gerkema et al., 1990; Cohen and Albers, 1991; Schwartz and Zimmerman, 1991). Numerous other lines of investigation have provided compelling support for the hypothesis that the SCN is a circadian oscillator (see Turek, 1985; Meijer and Rietveld. 1989). More recently, several laboratories have shown that transplanted fetal or neonatal $\mathrm{AH}$ tissue containing the $\mathrm{SCN}$ is capable of restoring a circadian rhythm of activity to rodents rendered arhythmic by SCN destruction (Sawaki et al., 1984; Lehman et al., 1987; DeCoursey and Buggy, 1988; Ralph et al., 1990; Boer and Griffioen, 1990; Saitoh et al., 1991). The success of these experiments has offered an opportunity to gain insight into the extent to which the oscillator located in the SCN is responsible for generating a fundamental characteristic of circadian behavior, i.e., the species- or straintypical $\tau\left(\tau_{\text {species }}\right)$ first noted by Pittendrigh and Daan (1976).

Simple restoration of rhythmicity to an arhythmic host by implantation of putative oscillatory tissue is not sufficient to establish the donor tissue as a circadian pacemaker or even as a circadian oscillator. Rather than having an oscillatory capacity, the transplanted tissue may merely play a permissive role allowing a host circadian oscillator to be expressed. Hamster homograft experiments have, indeed, resulted in an apparent conservation of $\tau_{\text {spccics }}$ (Lehman et al,, 1987; DeCoursey and Buggy, 1988), while rat homograft experiments have done so inconsistently (Sawaki et al., 1984; Boer and Griffioen, 1990; Griffioen et al., 1993). Yet while homograft experiments have provided useful information about the necessity of the SCN for the expression of circadian rhythmicity, they are unable to address the capacity of the SCN to generate and/or determine the resultant speciestypical circadian period. Because the host and donor have the same $\tau_{\text {species }}$, the resultant behavior can be ascribed to either the donor or host. It is, therefore, essential that a primary characteristic of the donor circadian rhythm (such as period or phase) be transferred and subsequently identified in the restored circadian activity of the host (Zimmerman and Menaker, 1979).

Moreover, in determining pacemaker function (i.e., in estab- 
lishing that the implanted tissue contains either an autonomous or even a hierarchically dominant oscillator), the transference of period alone is an insufficient measure in homograft or allograft preparations. In his seminal work using transplantation methods to identify the locus of the cockroach circadian pacemaker, Page (1982) was able to identify donor-typical periods following optic lobe transplantation between individuals raised under different lighting conditions. However, a second experiment was necessary to establish that the optic lobes were, in fact, the site of the circadian pacemaker in the cockroach. In the cockroach, a neural connection between the optic lobe(s) and the midbrain is required for the expression of circadian rhythmicity (Page, 1978). Considering the possibility that only the optic lobe component of the cockroach circadian system was effected by the lighting conditions under which the animals were raised, Page had undertaken the additional step of specific cooling of the midbrain to shift the potential oscillator in that structure. The observation that this manipulation had no effect on overt rhythmicity (Page, 1981), taken together with the results of the transplantation experiments, provided the compelling evidence that the pacemaker was located within the optic lobe itself.

Thus, because the rest of the host brain in the cockroach transplantation experiments was not necessarily different (even though the periods expressed by the intact animals were markedly different), the transplantation technique could identify a hierarchically dominant oscillator or circadian pacemaker in the optic lobes only after the potential contribution of the rest of the brain was thoroughly removed. However, transplantation of neural tissue from one donor species into a host of a different species offers a paradigm in which the hosts are necessarily different if the donor and host have behaviors that can be reliably identified as species specific. Thus, in determining the locus responsible for generating the differences between species-typical $\tau s$, the SCN-lesioned hamster CNS is inherently different from the milieu usually encountered by the anterior hypothalamic oscillator of the mouse or the rat.

The use of cross-species transplantation opens a variety of new approaches to questions not readily accessible through other methods. Balaban and colleagues (1988) were the first to use the ncural heterograft technique successfully to examine the transference of species-typical behavior; quail donor-chick host chimeras, which had received quail mesencephalic and diencephalic primordium, demonstrated quail-typical crowing behavior. Moreover, unlike the use of homografts, cross-species neural implants can exploit the full potential of transplantation techniques for investigating the neural regulation of behavior through unambiguous demonstration of graft/host integration concomitant with donor-derived recovery of function (Klassen and Lund, 1988; Saitoh et al., 1991; Wictorin et al., 1991; Sollars and Pickard, 1993, 1994).

To determine the direct relationship between restored circadian behavior attributable specifically to the donor tissue, and additionally to describe the full extent of donor integration with the host, heterografts of AH tissue were conducted. The use of donors from three rodent species with significantly different species-typical $\tau s$, makes it possible to determine the extent to which this region alone is responsible for generating the species differences in the circadian period of wheel-running behavior. Moreover, the availability of species-specific antibodies provides a means of examining the full extent to which the implant establishes neuronal integration with the host.

\section{Materials and Methods}

Three series of fetal anterior hypothalamic transplantation experiments were conducted. Male hamsters (Mesocricetus auratus), received from Charles River Laboratories [LAK:LVG (SYR)] at 6 weeks of age served as transplant tissue recipients in all experiments. Hamsters were housed individually in cages equipped with running wheels in light-tight, ventilated chambers (six cages/chamber) with food and water freely available. Wheel-running activity was recorded on Esterline 20-channel event recorders throughout the experiments as previously described (Pickard et al., 1987). All animals were maintained in constant illumination conditions consisting of either constant dark (DD), constant dim red light (RR) (approximately 1 lux), or constant light (I.I.) (approximately $10 \mathrm{lux}$ ). After recording baseline wheel-running activity, bilateral radiofrequency lesions were aimed at the $\mathrm{SCN}$ of each hamster as previously described (Sollars and Pickard, 1993). Animals demonstrating several weeks of disrupted circadian activity rhythms were implanted with fetal AH tissue from (1) fetal hamster donors (E13-E14, hamster homografts), (2) fetal mouse donors (E15-E17, C57BL/6J mouse heterografts), or (3) fetal rat donors (E15-E17, Sprague-Dawley rat heterografts).

Implantation surgery was performed as previously described (Sollars and Pickard, 1993). Briefly, pregnant animals were decapitated and fetuses removed by Caesarean section. Each fetus in its amniotic sac and with the placenta still attached was placed in a chilled, sterile buffered salt solution. When all of the fetuses were removed, the first host hamster was positioned in a Kopf stereotaxic apparatus. After the skull was exposed, a Wiretrol micropipette was positioned over one of the holes previously drilled for the lesion electrode and the hole was reopened. At this point, one or two fetuses were removed from the culture medium and decapitated. The brain of each fetus was removed and placed in sterile culture medium. Tissue for implantation was dissected from the base of the brain by four angled microincisions placed through the optic chiasm, rostral to the median eminence, and through the lateral tuberal region on each side. This yielded a pyramidal piece of hypothalamic tissue with a base approximately $1-1.5 \mathrm{~mm}$ on a side. (Control preoptic area tissue, lacking the SCN, was dissected to be of similar size, but the caudal-most incision was through the optic chiasm.) The block(s) of tissue for implantation were then transferred to fresh, sterile culture medium, drawn up into a sterile glass micropipette, stereotaxically lowered, and injected into the third ventricle of the host brain. The micropipette was left in place for $1 \mathrm{~min}$, and was then slowly retracted with slight positive pressure on the syringe plunger to minimize the movement of the implant up the micropipette track. The time that elapsed between an individual donor's removal from the uterus and its tissue implantation was never greater than $90 \mathrm{~min}$. Typically, five hosts received implants from one litter.

Immediately after surgery, each heterograft host received an intraperitoneal injection of cyclosporin A (CsA, Sandimmune, Sandoz Pharmaceutical, $10 \mathrm{mg} / \mathrm{kg}$ ). Daily injections were administered for up to 10 $\mathrm{d}$ at irregular clock times to minimize the possible synchronizing effects of the injections. Although the full benefits of CsA are not obtained unless it is administered throughout the expected survival time, the injections were sufficiently noxious to cause a suppression of wheelrunning behavior in the hamster. Therefore, the injections were stopped after $10 \mathrm{~d}$, or less for any individual animal whose recovery appeared to be in jeopardy (as judged by lethargy and low water intake). Although all of the animals recovered from the surgery itself, one animal died within 1 week of the implantation. Following implantation, the wheel-running activity of all animals was monitored continuously for a period ranging from 6 to approximately 30 wccks.

Behavioral data analysis was conducted using a computer-based quantification system developed in our laboratory. Using a Bioquant and Optimas image processing system integrated with an IBM PS/2 50Z computer, unfiltered wheel-running activity records were digitized in 3 min bins and stored. The record for each animal was then analyzed for the presence of periodicity, and $\tau$ was determined, according to the $\chi^{2}$ periodogram method of Sokolove and Bushell (1978). In order to analyze the continuous record (several months of data), sequential evaluations were made of the periodicity within $21 \mathrm{~d}$ intervals, advancing in $7 \mathrm{~d}$ increments. The overlapping evaluations demonstrated the emergence of rhythmicity and gave an assessment of the duration of any rhythms that emerged at irregular intervals following the implantation.

When applying the $\chi^{2}$ analysis to circadian rhythms, Sokolove and Bushell (1978) describe two types of error [i.e., misidentification of a peak as significant (type I), and failure to identify a significant periodic 
component (type II)], which tend to be inversely present in a given application. However, according to one of the authors (Sokolove, personal communication), the most prevalent error arising when the $\chi^{2}$ periodogram analysis is applied to real data is the type I error. As a result, significant periodicity was recognized in the current analysis only when it reached a level of significance of $p<10^{-6}$. It should be noted that the $Q_{P}$ values (indicating the strength of the periodicity) for intact rhythms are typically greater than 200 , whereas the critical value for $\alpha$ $=10^{-6}$ for circadian periods is approximately 70 , and each order of magnitude change in $\alpha$ varies the critical value by approximately 6 units. Furthermore, a stable restoration of rhythmicity was recognized only when significant periodicity was evident for at least two consecutive 21 -d intervals, and the measured values for $\tau$ did not differ by more than $0.50 \mathrm{hr}$.

For each animal demonstrating significant peaks of restored rhythmicity, the average period over the course of the experiment was used as the value for the restored period for the individual. Values grouped according to donor species were compared both to the values for intact control animals of the same species and of the host species, and to the values for the grafts of the other donor species. The data were analyzed for statistical significance with an analysis of variance (ANOVA). Post hoc analysis was performed using Duncan's multiple range tests with harmonic means. The Bonferroni adjustment for multiple comparisons was applied, and an overall experimental alpha level of no greater than 0.05 was preserved.

At the termination of behavioral data collection, all animals received bilateral intraocular injections of horseradish peroxidase (HRP) or HRP conjugated to cholera toxin $\beta$-subunit (CT-HRP) generously provided by Dr. Richard Miselis, University of Pennsylvania. Tissue was prepared for both HRP histochemistry and immunocytochemical analysis. Histochemical demonstration of anterogradely transported HRP in retinal terminals over the SCN region was performed as described previously (Pickard and Silverman, 1981) except that 4\% paraformaldehyde was used as the fixative for compatibility with immunocytochemistry. Analysis of CT-HRP labeling of the RHT in intact animals demonstrated that this fixative resulted in no substantial diminution in label in the SCN. Outgrowth of heterograft neuronal processes into the host hypothalamic neuropil was demonstrated immunocytochemically using species-specific monoclonal antibodies. M-6, a monoclonal antibody specific for a surface glycoprotein on mouse neurons (Lund et al., 1985) was generously supplied by Dr. Carl Lagenaur, University of Pittsburgh. RMO108, a monoclonal antibody generated against rat neurofilament (Lee et al., 1987) that recognizes both rat and mouse but not hamster neural tissue, was kindly supplied by Dr. Virginia Lee, University of Pennsylvania. Immunocytochemical analysis on alternate $24 \mu \mathrm{m}$ coronal sections cut on a cryostat was conducted as described previously (Sollars and Pickard, 1993).

\section{Results}

\section{Hamster homografts}

Bilateral lesions ained at the SCN were conducted in 18 hamsters maintained in wheel-running cages in dim RR conditions throughout the experiment. Six animals remained rhythmic following the lesioning and were removed from the study. Lesions aimed at the SCN in the remaining 12 animals resulted in the disruption of circadian wheel-running activity in all animals (Fig. 1). These animals received $\mathrm{AH}$ homografts from fetal donors approximately 6 weeks after SCN lesioning. Following the fetal AH implantation surgery, animals' wheel-running behavior was monitored for an additional 14 weeks. At that time, each animal received binocular injections of CT-HRP and every third section through the hypothalamus was processed for HRP histochemistry.

Of the 12 implanted animals, SCN lesion evaluation, aided by CT-HRP histochemical analysis of anterogradely labeled retinohypothalamic fibers and terminals, indicated complete SCN destruction in all 12 animals. Ten of these animals demonstrated a restoration of circadian rhythmicity, which was evident approximately 2-5 weeks after implantation surgery (Fig. 1). In some animals, the restored rhythm became apparent somewhat abruptly (Fig. 1a), whereas in others, rhythmicity was manifest more gradually (Fig. $1 b$ ).

The average period of the restored circadian rhythm, based on periodogram analysis, was $24.01 \pm 0.06 \mathrm{hr}$ (mean $\pm \mathrm{SEM}$, $n=10$ ), which was not significantly different from the mean period of a group of intact control hamsters maintained in DD for several weeks $(24.07 \pm 0.01 \mathrm{hr}, n=11 ; p>0.5)$. A threedimensional display of the quantitative periodogram analysis of the behavioral data presented in Figure $1 b$, is illustrated in Figure 2. Peaks that emerge above the superimposed plane meet the established criterion for statistical significance as described above. The statistical display demonstrates an initial periodicity in the circadian range $(\tau \approx 24.05 \mathrm{hr}$ ) followed by several weeks postlesioning, during which no significant periodicity is evident. This arhythmic period is followed by the reestablishment of strong circadian periodicity following fetal AH implantation (mean $\tau=24.16 \mathrm{hr}$ over weeks $\approx 10-22$, Fig. 2 ).

Histological examination revealed that all of the implanted hamsters had large, well-vascularized grafts in the third ventricle which, in some cases, was filled by the implant. Neither of the ineffective implants was grossly discernable histologically from those that were effective in restoring behavioral rhythmicity, and both were positioned in the third ventricle near the site of the lesion. All of the implants had regions in which the ependymal lining of the host third ventricle was not evident; in these regions the implant seemed to merge directly with the host brain. A cellpoor region was also a characteristic element in most of the areas in which the graft/host interface was not defined by the ventricular wall and may indicate the limit of the graft cell bodies in these areas. Graft/host interconnections were not further assessed in the homografts due to the inherent difficulty in distinguishing implant derived fibers from those of the host. Graft/host integration is addressed below in the AH heterografts.

\section{Mouse heterografts}

A series of three mouse-to-hamster heterograft experiments were conducted with illumination conditions and analyses varying slightly among experiments. In the first experiment $(n=47)$, hamsters in wheel-running cages were maintained throughout under dim LL (10 lux) conditions and all animals were implanted with fetal mouse AH tissue. In the second $(n=30)$ and third $(n=5)$ experiments, hamsters were housed throughout in dim RR ( 1 lux) and animals not rendered arhythmic by the lesions aimed at the SCN were removed from the study. In the first and second experiments, each $\mathrm{SCN}$-lesioned animal received a single fetal mouse AH implant and the M-6 mouse-specific monoclonal antibody was used for immunocytochemical evaluation of implant outgrowth. In the third experiment, each SCN-lesioned hamster received fetal $\mathrm{AH}$ tissue taken from multiple donors and the RMOIO8 monoclonal antibody was used for immunocytochemical analysis. The results described below are a compilation of the threc independent experiments and are summarized in Figure 3.

Wheel-running activity was recorded in 82 hamsters: 69 received lesions aimed at the SCN and 13 served as unlesioned controls. Of the $69 \mathrm{SCNx}$ animals, 47 were implanted with fetal mouse $\mathrm{AH}$ tissue, 10 received fetal mouse preoptic area implants, and 12 were removed from the study after SCN-lesioning failed to abolish the circadian activity rhythm. One of the AHimplanted animals died prior to histological evaluation; this animal's behavioral data were not included in the analysis. Lesion assessment in the 46 arhythmic AH-implanted hosts, aided by 
a

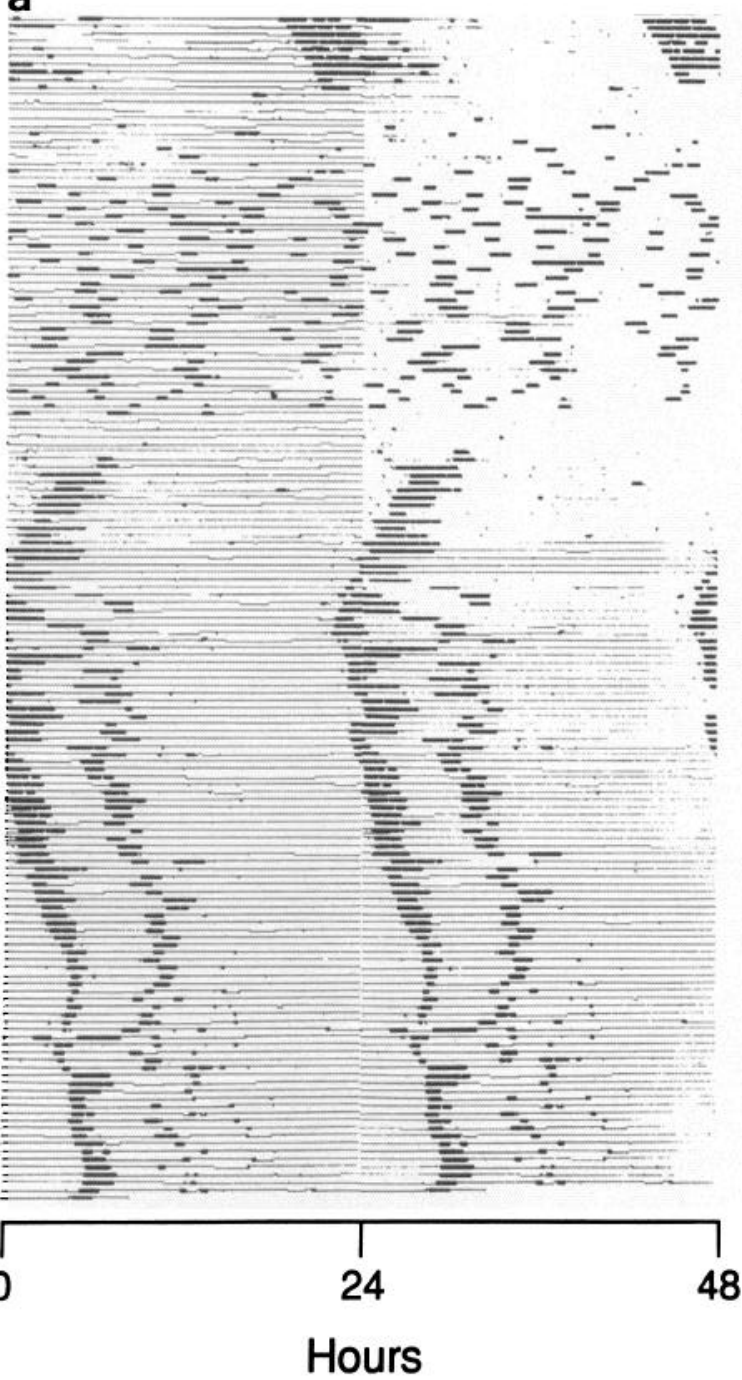

b

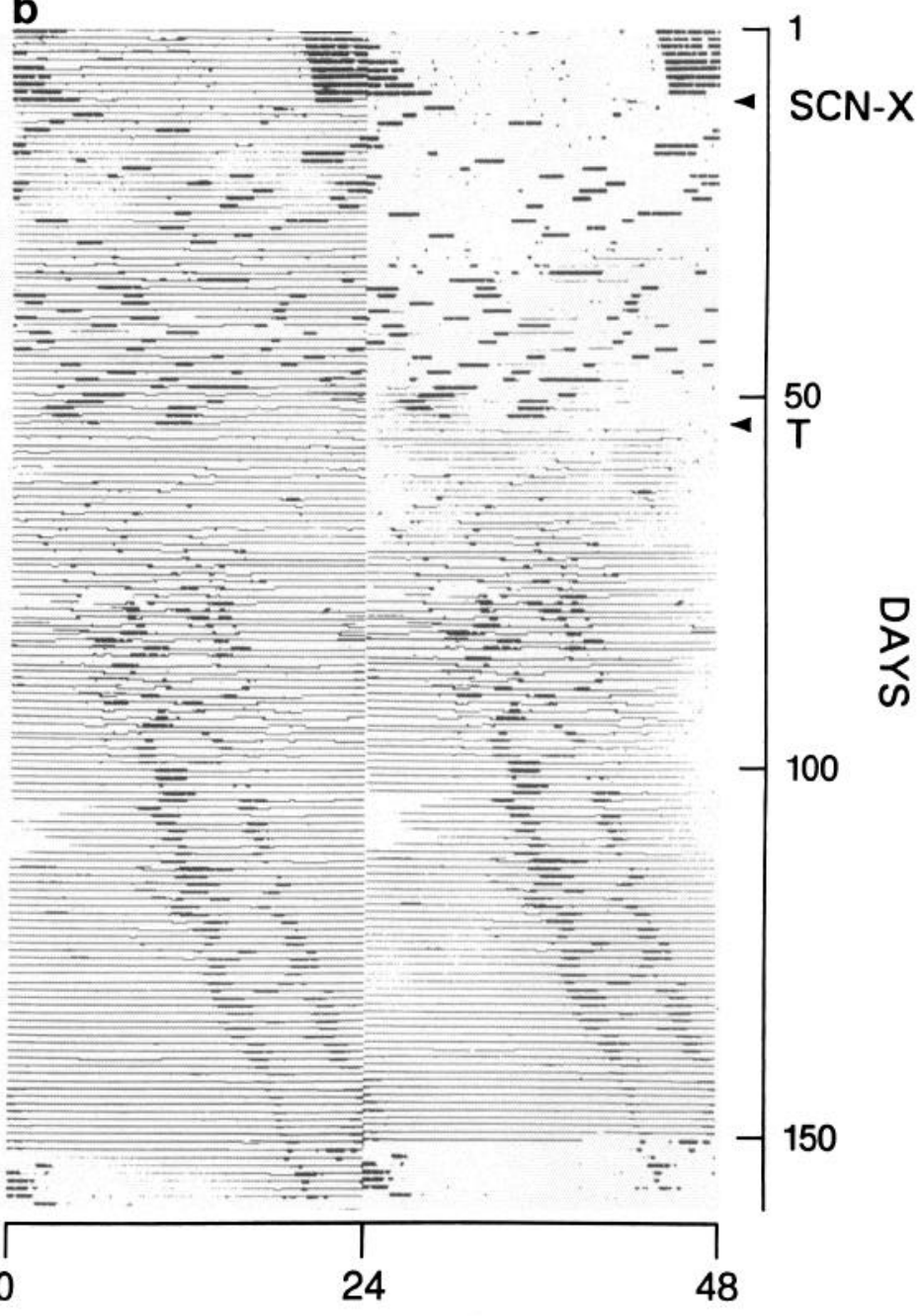

Figure 1. Wheel-running activity records for two hamsters bearing $\mathrm{AH}$ homografts. Animals were maintained in constant dim red light conditions throughout the experiment. On the day indicated by $S C N-X$, each animal received bilateral lesions that completely ablated the SCN. Approximately 6 weeks after SCN lesioning, each hamster received an AH transplant ( $T)$ from a fetal hamster donor. A restored circadian pattern of wheel-running activity is evident in both animals; restored rhythmicity is apparent approximately $10 \mathrm{~d}$ after AH implantation in one animal (a), whereas it appears more gradually in the other $(b)$. The mean period of the steady-state circadian rhythm in each case was hamster-typical $(a=24.08$ hr and $b=$ $24.16 \mathrm{hr}$ ). Activity records are double plotted for ease of visual inspection.

HRP histochemical detection of labeled retinal terminals in the host $\mathrm{SCN}$, revealed 22 to have complete $\mathrm{SCN}$ destruction. The behavioral data of any animal with remnant host SCN were not included in the analysis of the restored periodicity, even if the behavioral patterns prior to the implant surgeries were determined to be completely arhythmic. This exclusion was necessary in order to attribute the restored rhythmicity to the implanted tissue alone.

Fifteen of the 22 complete SCNx animals were judged to have viable mouse implants at the termination of the experiment; 9 of these animals demonstrated restoration of circadian rhythmicity (Figs. 4, 5). The mean period of the restored circadian activity rhythms of hamsters bearing mouse $\mathrm{AH}$ implants was $23.47 \mathrm{hr}$, similar to the $\tau_{\mathrm{DD}}$ of a group of 23 intact C57BL/6J mice whose wheel-running behavior was monitored over 6-8 weeks in constant dark $(23.47 \pm 0.08, n=9$ vs $23.43 \pm 0.02$, $n=23 ; p>0.5$ ). The mean $\tau$ for each animal was determined from quantitative periodogram analysis; Figure 6 provides a display of the periodogram analysis of the behavior illustrated in Figure 5. The efficacy of viable mouse heterografts in restoring rhythmicity to SCNx hamster hosts was $60 \%(9 / 15)$, somewhat less than the efficacy of the hamster homografts conducted in our laboratory $(83 \% ; 10 / 12)$. However, the overall success rate of mouse heterograft rhythm restoration was only $40 \%(9 / 22)$, a difference accounted for by graft rejection.

The emergence of restoration of the circadian wheel-running activity in the mouse heterografts was slightly delayed compared to the hamster homografts. Restored circadian activity in mouse heterografts became evident 2-12 weeks postimplantation, whereas hamster homografts restored rhythmicity within 5 weeks in all 10 cases. Restored heterograft rhythms could, nevertheless, be quite stable, as evident in Figure 4; the renewed rhythm in this case was still unmistakable 7 months after the fetal mouse $\mathrm{AH}$ tissue was transplanted into the hamster host. 

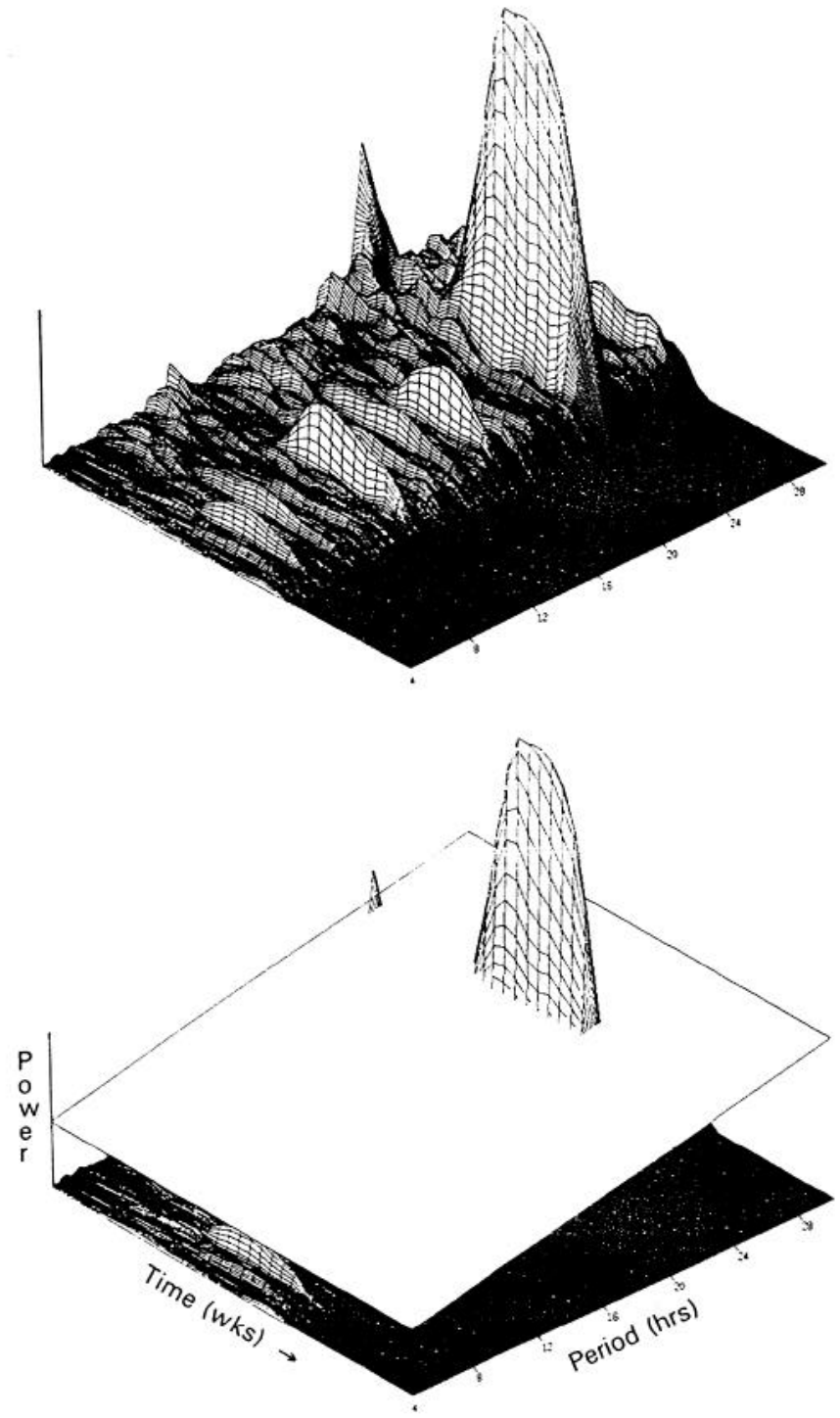

Figure 2. A three-dimensional display of a $\chi^{2}$ periodogram analysis of the wheel-running activity data of the hamster bearing an $\mathrm{AH}$ homograft illustrated in Figure $1 b$. Sequential evaluations were made of periodicity within $21 \mathrm{~d}$ intervals, advancing in $7 \mathrm{~d}$ increments. Robust periodicity is reflected by large amplitude peaks, plotted as power (upper panel). Peaks emerging above the superimposed plane indicate significant peaks at $p<1 \times 10^{-6}$ (lower panel). The initial peak indicating periodicity in the circadian range prior to $S C N$ lesioning $(\tau \approx 24.05 \mathrm{hr})$ appears truncated because only $10 \mathrm{~d}$ of prelesion data were collected and disappears following SCN destruction. A highly significant peak $(\tau$ $=24.16 \mathrm{hr}$ ) reappears following $\mathrm{AH}$ transplantation, and persists until the termination of the experiment, indicating restored behavioral rhythmicity. For details, see Materials and Methods.

The SCN-lesioned hamsters that received fetal preoptic area implants as a control for the specificity of $\mathrm{AH}$ implants in rhythm restoration $(n=10)$ remained arhythmic to the termination of the experiment 10 weeks following implantation. In addition, there was no change in the activity of any of the unlesioned hamsters $(n=13)$ that received fetal mouse AH tissue implanted into the third ventricle, other than an occasional shortening of $\tau$ (5-24 min in 5/13 animals) immediately following the implantation surgery. This was interpreted to have been the result of physical damage to the $\mathrm{SCN}$ during the implantation procedure, since there was no correlation between a slight

\section{Summary of Mouse AH Heterografts}

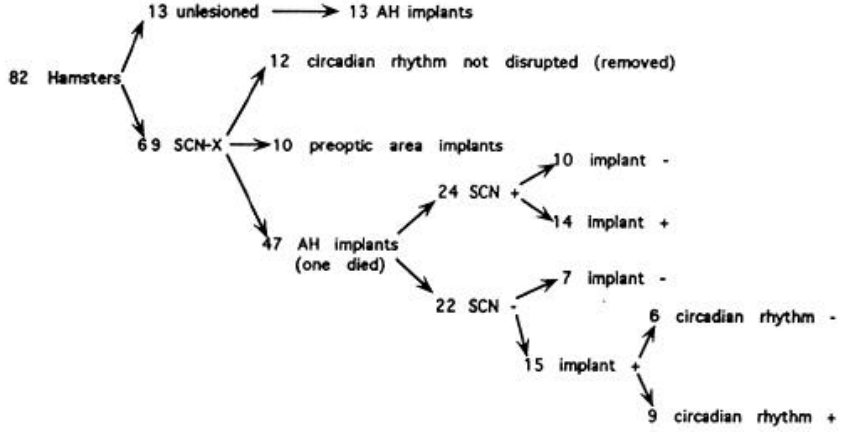

Figure 3. Summary of mouse AH heterografts. Of $69 \mathrm{SCN}$-lesioned hamsters, 47 received fetal mouse $\mathrm{AH}$ implants. Histological analysis indicated that 22 of these hamsters had complete SCN destruction $(S C N-)$, whereas 24 had at least some host $S C N$ remaining $(S C N+)$; these latter animals were not considered further in the analysis. Fifteen of the $22(\mathrm{SCN}-)$ animals contained viable mouse implants at the termination of behavioral data collection. Of these 15 animals, 9 demonstrated restored circadian rhythmicity as determined by $\chi^{2}$ analysis.

change in period and the presence of an implant in the host at the termination of the experiment.

Mouse $\mathrm{AH}$ implants in the hamster third ventricle and fiber outgrowth into the adjacent hypothalamic neuropil were clearly identified immunocytochemically in experiments 1 and 2 with the M-6 monoclonal antibody that recognizes a cell surface glycoprotein found on neurons of the mouse CNS (Lund et al., 1985). The implants were intensely stained (Fig. 7) and immunopositive fibers were evident leaving the implant and coursing in a ventrolateral direction through the host. The photomicrograph presented in Figure $8 a$ is of the implant of the animal with a restored activity rhythm illustrated in Figure 5. This AH heterograft from experiment 3 has been stained immunopositive with the RMO108 monoclonal antibody that recognizes mouse (and rat) but not hamster neurons (see Sollars and Pickard, 1993). The implant in the third ventricle is intensely stained and several fibers that emerged from the implant rostral to the plane of this section are apparent, coursing through the periventricular zone of the host hypothalamus parallel to the implant. The extent of implant/host integration diminished rapidly with distance from the implant. No apparent difference was discernable in the pattern of implant-to-host integration between implants that did $(n=9)$ and those that did not $(n=6)$ restore circadian activity to hamsters with complete SCN ablation.

\section{Rat heterografts}

Two independent rat-to-hamster heterograft experiments were performed. The first ( $n=7 \mathrm{SCN}$-lesioned animals) was conducted under $\mathrm{LL}_{10}$ lighting conditions. In the second experiment, 60 hamsters received lesions aimed at the SCN and were maintained in dim RR conditions throughout. In the following description of the rat heterograft findings, the results for the two experiments have been combined and are summarized in Figure 9. Five to seven weeks after SCN lesioning, animals demonstrating disrupted circadian activity rhythms $(n=45)$ received rat AH heterografts from either a single donor $(n=20$, including all animals in experiment 1$)$ or two donors $(n=25)$. Five months after implantation, animals were injected binocularly with HRP (experiment 1) or CT-HRP (experiment 2). Tissue was 


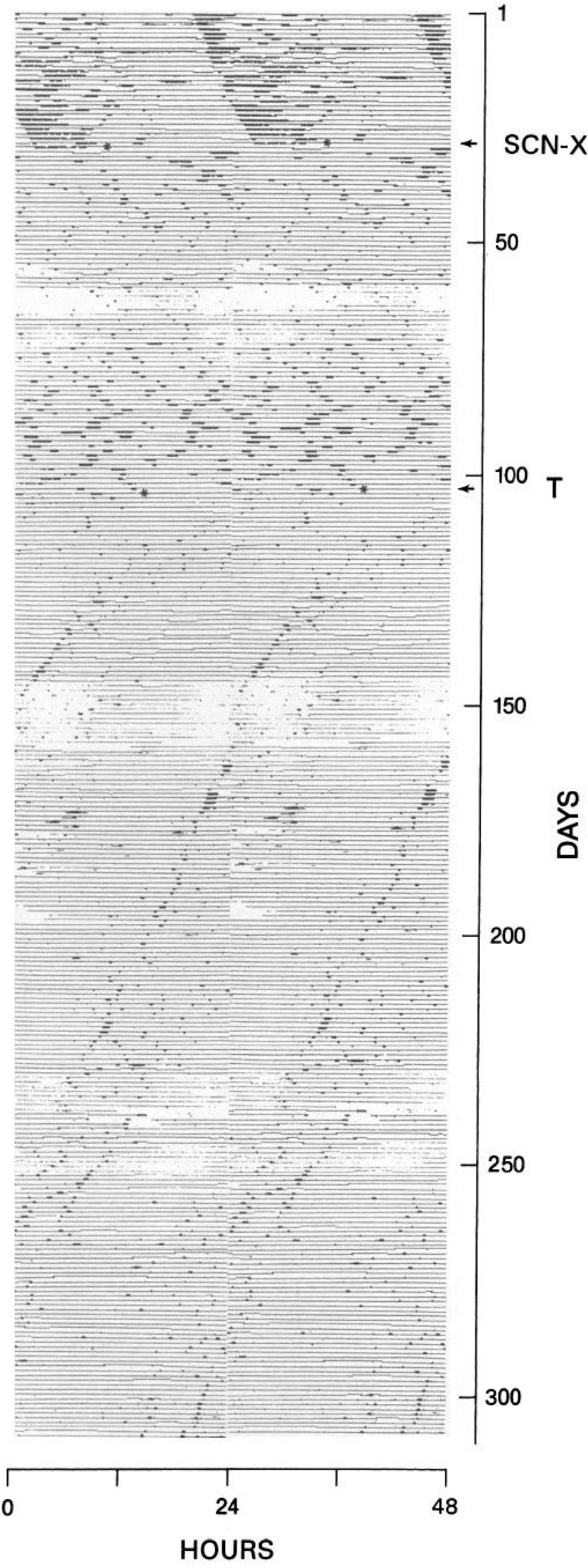

Figure 4. Wheel-running activity record for a hamster bearing a mouse $\mathrm{AH}$ heterograft. The hamster was maintained in constant dim processed for HRP histochemistry and immunocytochemical analysis. Implant integration with the host was evaluated using the RMO108 monoclonal antibody that recognizes rat (and mouse) but not hamster nervous tissue.

SCN lesion evaluation, aided by the analysis of labeled retinal processes at the site of the lesion, indicated that 34 of the 45 hamsters that had received rat $\mathrm{AH}$ implants also had complete SCN destruction. Of these animals, 29 had viable rat grafts in the third ventricle and 17 of these animals had exhibited a restoration of circadian rhythmicity (see Figs. 10, 12). The mean $\tau$ of the restored circadian rhythms observed in the rat-to-hamster experiments was $23.60 \pm 0.07(n=17)$. The emergence of restored rhythmicity for rat $\mathrm{AH}$ heterografts tended to occur slightly later than that for mouse $\mathrm{AH}$ heterografts, although some restored rhythms were evident within 2 weeks of the AH transplantation and the range of onsets (2-13 weeks postimplantation) was overlapping with that of the mouse heterografts. A three-dimensional display of the periodogram analysis of the behavioral data illustrated in Figure $10 a$ is presented in Figure 11.

The mean $\tau$ of the circadian rhythms restored following rat AH transplantation $(23.60 \pm 0.07 ; n=17)$ is significantly different from the mean $\tau$ of intact rats maintained in DD conditions for several weeks $(24.34 \pm 0.04 ; n=12, p<0.005)$. The $\tau$ of rat heterograft restorations also differs significantly from the restored period expressed in the hamster homograft experiment $(23.60 \pm 0.07$ vs $24.01 \pm 0.06, p<0.02)$. However, it does not differ significantly from the period restored by the mouse heterografts $(23.60 \pm 0.07$ vs $23.47 \pm 0.08, p>0.5$; compare Figs. 4, 5 with Fig. $10 a, b)$. It is interesting to note, however, that a single rat $\mathrm{AH}$ heterograft did restore rhythmicity to the SCN-lesioned hamster with a $\tau$ approximating that of the intact control group; the mean period length of the restored rhythm in this case was $24.24 \mathrm{hr}$ (Fig. 12). The implant for this animal was among the larger implants observed, measuring over $600 \mu \mathrm{m}$ in its mediolateral extent, and its greatest volume was situated caudally in the host brain, near the arcuate nucleus, but in neither of these respects was it unique among the rat $\mathrm{AH}$ implants. Thus, we found no anatomically based explanation for the behavioral difference observed in this individual case. Table 1 summarizes all the behavioral restorations.

The overall success rate of restorations by rat heterografts in complete SCN-lesioned hamsters was 50\% (17/34; Fig. 9), slightly greater than the $40 \%$ success rate of mouse heterografts (9/22; Fig. 3). However, the viability of rat heterografts (independent of host SCN status) was also slightly greater than that noted for mouse AH implants ( $80 \%$ vs $54 \%$, respectively). Accordingly, a comparison of the efficacy of viable rat heterografts to restore rhythmicity to complete $\mathrm{SCN}$-lesioned hamsters with the efficacy of viable mouse heterografts to restore rhythmicity to such hamsters reveals the similarity between the species (17/ 29 or $59 \%$ for rat and $9 / 15$ or $60 \%$ for mouse heterografts; see Figs, 3, 9).

The surviving rat $\mathrm{AH}$ implants appeared generally more robust than the mouse $\mathrm{AH}$ heterografts, and appeared to flourish in the foreign environment of the hamster CNS (Fig. 8b). They

$\leftarrow$

red light throughout the 10 month experiment. On the day indicated by $S C N-X$ and at the time indicated by *, the SCN were bilaterally lesioned. Approximately 10 weeks following $\mathrm{SCN}-\mathrm{X}$, the hamster received a fetal mouse AH transplant $(T)$. Circadian rhythmicity reemerges 3-4 weeks later and persists to the termination of the experiment. 


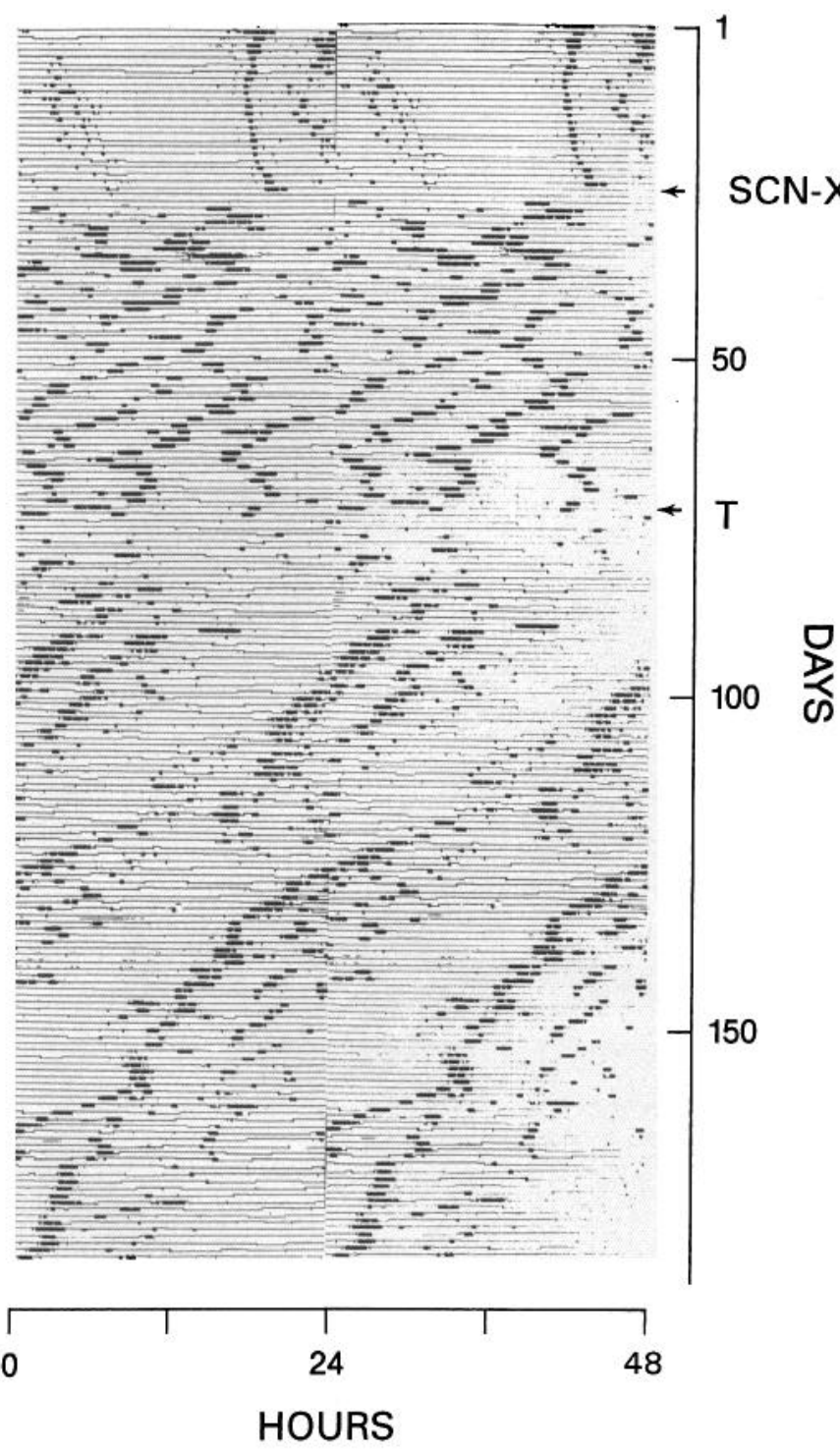

Figure 5. Wheel-running activity record for a hamster bearing a mouse AH heterograft. $S C N-X$ indicates day of SCN lesioning. $T$ indicates day of fetal mouse $\mathrm{AH}$ transplantation.

demonstrated less necrosis than mouse $\mathrm{AH}$ implants, were more fully vascularized, and were consistently much larger, sometimes filling the entire third ventricle (Fig. 13). Indeed, prior to immunocytochemical analysis, several rat heterografts were distinguishable from homografts only by virtue of their size, dwarfing the homografts in comparison. Immunocytochemical staining with the anti-neurofilament antibody (RMO108) revealed extensive outgrowth of donor neuronal fibers into the host brain. Donor fibers were often observed at distances of up to $3 \mathrm{~mm}$ from the boundary of the implant. They frequently extended dorsally from the implant, coursing around the anterior commissure to its dorsal aspect and into the region of the bed nucleus of the stria terminalis. An example of such a pattern of outgrowth is provided in Figure 14. In many cases, the outgrowth from the implant projected primarily in a dorsolateral direction, and in all cases the graft/host integration diminished gradually with distance from the implant. The composite examination of the rat $\mathrm{AH}$ heterografts yielded no obvious differences between the rat

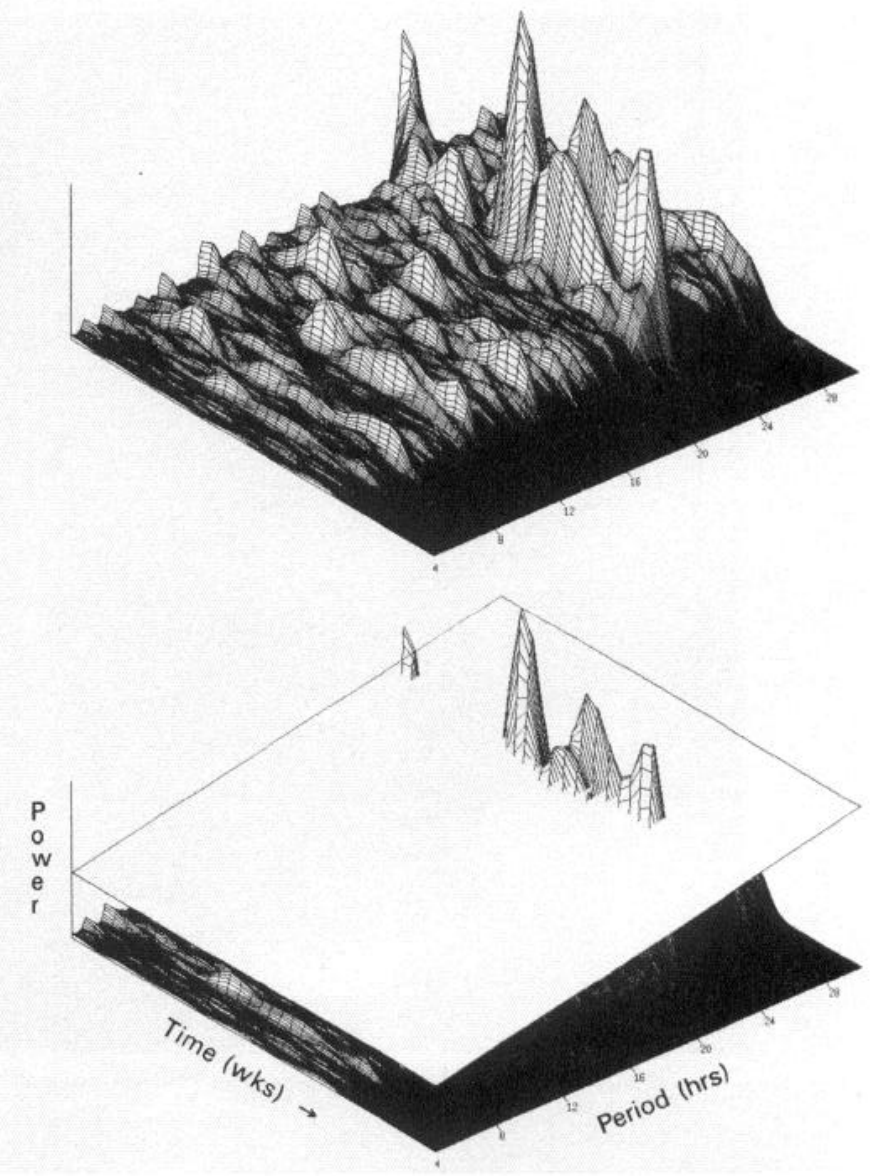

Figure 6. A three-dimensional display of a $\chi^{2}$ periodogram analysis of the wheel-running activity data of the hamster bearing a mouse $\mathrm{AH}$ heterograft illustrated in Figure 5 (see Fig. 2 for further detail). Initial circadian rhythmicity $(\tau \approx 24.1 \mathrm{hr}$ ) was disrupted following SCN destruction. A restored circadian rhythm with a mean period of $23.6 \mathrm{hr}$ is again evident following mouse $\mathrm{AH}$ transplantation.

heterografts that restored circadian rhythmicity and those that did not, either in the extent of implant integration with the host or in the pattern of fiber outgrowth into the host.

\section{Discussion}

To identify the SCN as a circadian pacemaker through the use of neural transplantation techniques, a primary characteristic of circadian behavior such as period must unequivocally accompany the transplanted tissue (Page, 1982; Sollars and Pickard, 1994). The AH heterograft experiments presented in this study were conducted in an attempt to transfer the species-typical circadian period of wheel-running behavior along with the implanted tissue. AH homograft experiments, although inevitably unable to address the fundamental question of whether the SCN functions as an autonomous mammalian circadian pacemaker, were useful in the present study to provide baseline information against which the heterograft experiments could be assessed. The SCN-lesioning and fetal $\mathrm{AH}$ implantation procedures employed in the present experiment yielded a successful restoration of rhythmicity in the majority $(83 \%)$ of the SCNx animals with AH homografts. Furthermore, the period of the restored rhythm following $\mathrm{AH}$ homograft implantation was not significantly different from the circadian period expressed by intact controls ( $24.01 \mathrm{hr}$ vs $24.07 \mathrm{hr}$ ), in agreement with the findings of the 

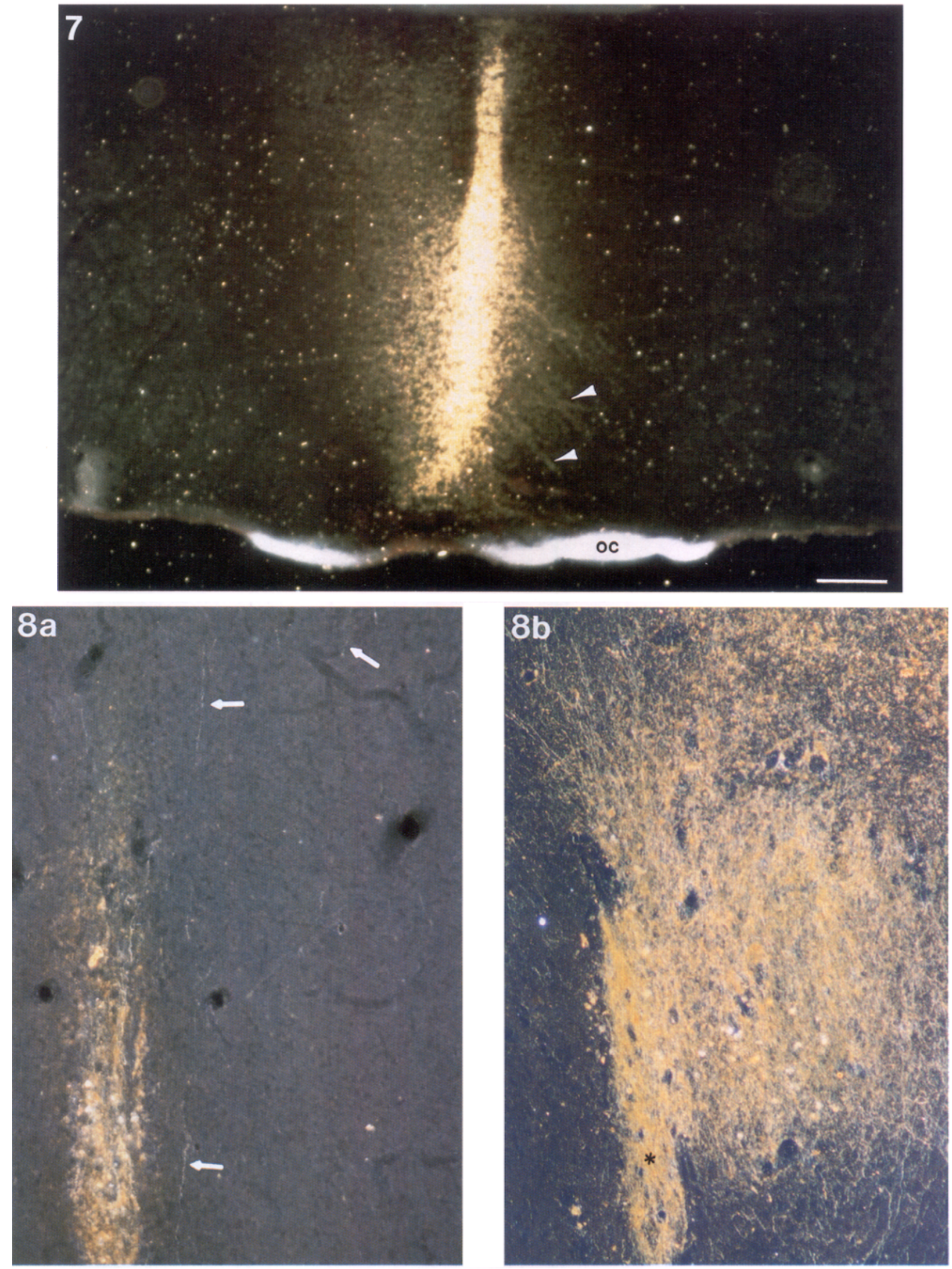

Figure 7. Dark-field photomicrograph of a coronal section through the anterior hypothalamus of a hamster with bilateral ablation of the SCN. Immunocytochemical staining with the mouse-specific M-6 monoclonal antibody demonstrates the presence of a mouse neuronal implant in the third ventricle of the host and the diffuse emanation of processes from the implant into the host periventricular zone. Additionally, discrete fascicles of mouse axonal processes (arrowheads) can be seen coursing ventrolaterally into the host hypothalamus. oc, optic chiasm. Scale bar, $250 \mu \mathrm{m}$. 


\section{Summary of Rat AH Heterografts}

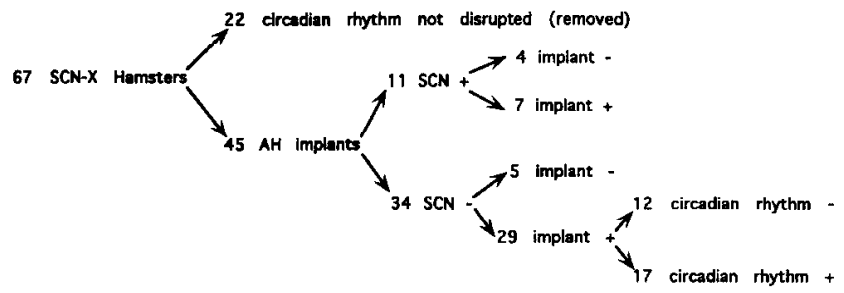

Figure 9. Summary of rat AH heterografts. Sixty-seven SCN-lesioned hamsters received fetal rat $\mathrm{AH}$ implants and 34 of these implanted animals were determined to have complete SCN ablation ( $S C N-$ ). Twenty-nine of these animals maintained a viable rat heterograft until the termination of the experiment and 17 of these animals demonstrated restoration of circadian rhythmicity.

earlier hamster homograft reports of Lehman et al. (1987) and DeCoursey and Buggy (1988).

In the present study, both mouse and rat AH heterografts were shown to be capable of restoring circadian rhythmicity to the majority of the SCNx hosts; $60 \%$ (26/44) of viable heterograft implants restored circadian rhythmicity. However, analysis of the circadian periods restored by the heterografts did not directly support the hypothesis that the implants contained a pacemaker solely responsible for generating the species-typical periods of each of the species used. Furthermore, because the restored periods were "species-typical" for only two of the three donor/ host combinations (i.e., hamster homografts and mouse-to-hamster heterografts), the results do not provide evidence of a consistent mechanism of period determination hetween these three species. It should be noted, however, that while the hamster homografts and mouse heterografts restored circadian rhythmicity with a $\tau$ similar to that of the intact donor species, the rat-tohamster heterografts imparted a period that was typical of neither the donor nor the host, but was not significantly different from the period expressed by the mouse heterografts.

There are several divergent interpretations of the differences between the restored periods described in this study. On one hand, based upon the observed data, it may be proposed that the generation of species-typical $\tau$ is, indeed, a property of the SCN of the hamster and the mouse, but is not a property of the isolated (transplanted) SCN of the rat. On the other hand, it may just as well be proposed that the generation of species-typical $\tau$ is not an inherent property of the SCN at all. For clarity, we will briefly discuss these two categories of interpretation under separate headings.

\section{Species-typical $\tau$ is a property of the SCN in some species}

This first proposition states that the generation of species-typical $\tau$ is a fundamental property of an oscillator located within the AII grafts donated by the hamster and the mouse, but not those of the rat. However, this is not an unequivocal assertion that under normal conditions (i.e., in the intact animal) the rat $\tau_{\text {species }}$ is not a fundamental property of the SCN. The general (but not universal) failure of the rat $\mathrm{AH}$ implant to restore the donortypical $\tau_{\text {speciss }}$ in transplantation paradigms may just as likely be due to a particular susceptibility of the rat SCN to damage or developmental disruption when subjected to the transplantation procedures.

However, at least one important caveat applies to this suggestion. The failure of the rat oscillator is not likely to be due to a general susceptibility to damage from the transplantation procedure. Such damage would have to be a widely variable factor, varying to some degree among individual implants within a given experiment, but also varying substantially among the numerous laboratories that have reported restoration of circadian rhythmicity following rat AH transplantation. Nevertheless, there has been remarkable consistency among the various reports of the periods restored by these implants (Sawaki et al., 1984; Kawamura and Nihonmatsu, 1985; Boer and Griffioen, 1990; Saitoh et al., 1990; Griffioen et al., 1993). Moreover, Saitoh and colleagues (1991), in an investigation of whether nocturnality or diurnality is conveyed through $\mathrm{AH}$ grafts, have reported that three of four rat heterografts in their rat-to-chipmunk model also expressed restored periods $<24 \mathrm{hr}$. In general, only a small percentage of the grafts within a given experiment have restored a "rat-typical" period in the range of $24.3 \mathrm{hr}$, while the rest have restored periods noticeably shorter than $24 \mathrm{hr}$. The consistency among the "atypical" periods is striking, suggesting that the lability of the oscillator would have to be a specific susceptibility (perhaps of a particular subset of cells within the oscillator) that results in the expression of a second preferred period in the range of $23.5 \mathrm{hr}$.

A second potential source of failure of the rat $\mathrm{AH}$ implant to restore species-typical rhythmicity might be a developmental time course for the establishment of $\tau_{\text {species }}$ in the rat lagging behind that of the mouse and hamster, or an arrest of development of the rat oscillator by the transplantation procedure. However, at least two points limit the strength of this suggestion. First, although the SCN of the rat has been demonstrated to have an oscillatory capacity by embryonic day 19 (Reppert and Schwartz, 1984), there is no evidence that varying the donor age between embryonic day 15 and postnatal day 2 has any lengthening effect on the restored period. Moreover, studies of the morphological development of the SCN within AH grafts have demonstrated an altered rate of development following transplantation procedures (Roberts et al., 1987; Boer and Grittioen, 1990; Griffioen et al., 1993), but have not suggested that development of the SCN is arrested at the point of transplantation, nor that it is slowed sufficiently to disrupt periodicity that emerges 4-6 weeks postimplantation.

Nevertheless, keeping these various caveats in mind, the combined AH transplantation data also support the suggestion that the differing efficacies of the AH grafts may be due to essential differences between the oscillatory mechanisms of the three rodent species used in these studies. Accordingly, while speciestypical $\tau$ may be a centralized property of both the hamster and mouse SCN, it may be suggested that the circadian pacemaker of the rat, responsible for generating its $\tau_{\text {species }}$, is a more distrib-

$\leftarrow$

Figure 8. Dark-field photomicrographs of the hypothalamus of hamsters bearing AH heterngrafts immunocytochemically stained with the RMO108 monoclonal antibody. $a$, Illustrates an immunostained mouse implant situated in the third ventricle of the host and several mouse neuronal processes coursing through the hamster hypothalamus (arrows). $b$, Illustrates the robust neuronal outgrowth typically observed from a rat AH implant (only part of which is represented in this photomicrograph by the *). Rat AH heterografts were larger, more fully vascularizcd and more extensively integrated with the hamster hypothalamus than were mouse AH heterografts. 


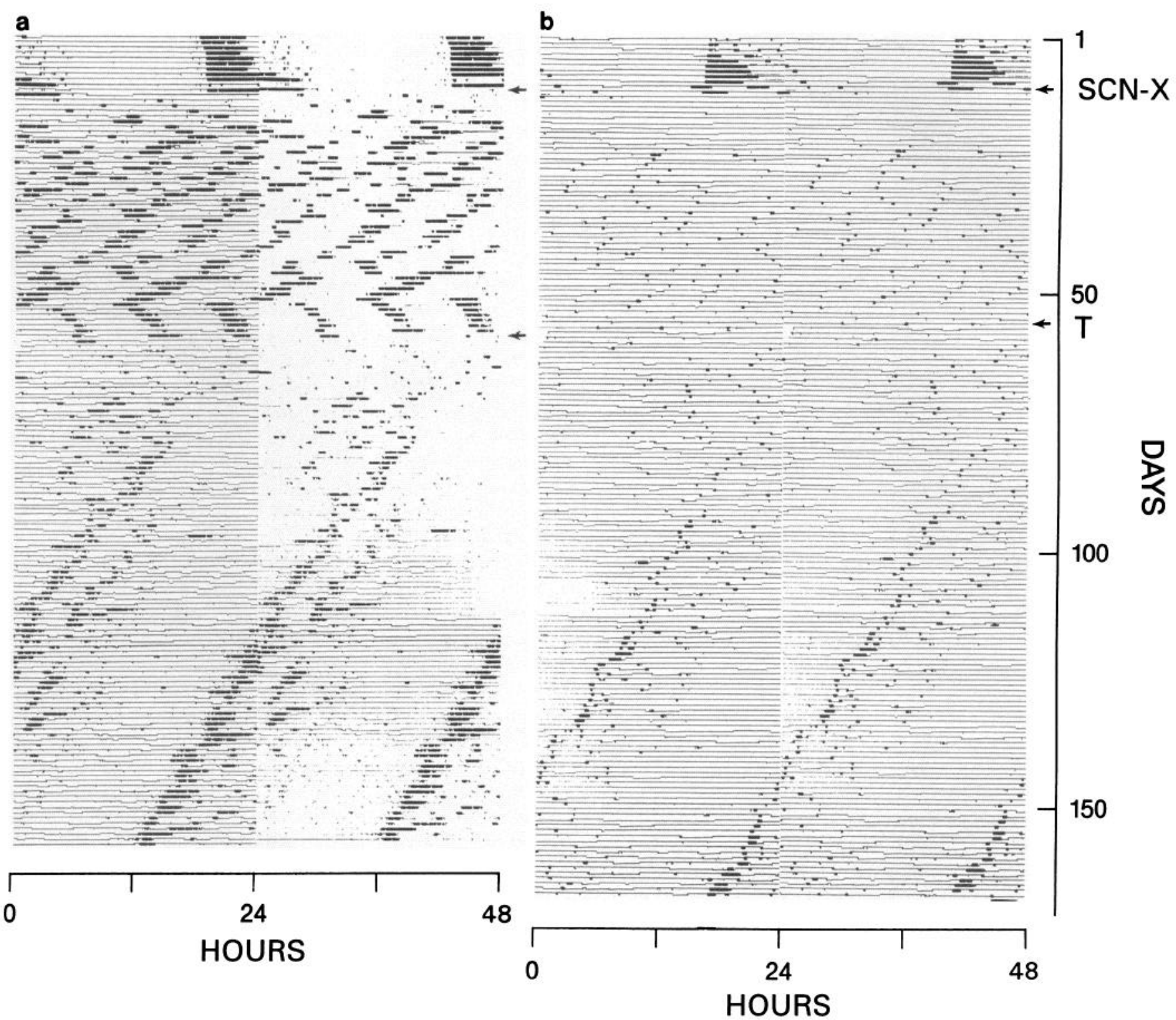

Figure 10. Wheel-running activity records for two hamsters $(a$ and $b$ ) bearing rat AH heterografts. $S C N-X$ indicates day of SCN lesioning. $T$ indicates day of fetal rat $\mathrm{AH}$ transplantation. The restoration of circadian wheel-running activity is clearly evident in both animals.

uted system. In further support of this suggestion, there is a variety of behavioral, anatomical, and physiological data indicating clear species differences between the circadian systems of the hamster and the rat (Rosenwasser, 1988).

\section{Species-typical $\tau$ is not a property of the $S C N$}

The second line of interpretation is in direct opposition to the first. It states that the generation of species-typical $\tau$ may not be a fundamental property of the SCN at all. On the contrary, the restored period of approximately $23.5 \mathrm{hr}$ may be a "heterografttypical" period determined by a remnant circadian pacemaker component in the SCN-lesioned hamster host, interacting differently with a heterograft $\mathrm{AH}$ implant than with an $\mathrm{AH}$ homograft. Since the SCN has long been known to be an oscillatory component of the circadian pacemaking system (see Turek, 1985), this, in turn, suggests that the endogenous hamster circadian pacemaker consists of a coupled multioscillatory system with at least one extra-SCN oscillator coupled to the component in the SCN.

This interpretation relies upon the notion that the SCN is a crucial link to the overt expression of circadian rhythmicity in the animal, and that $\mathrm{AH}$ heterografts somehow enable the preferential expression of the oscillator(s) normally muted by SCN ablation. There are several mechanisms by which this preferential expression of the extra-SCN oscillator(s) might occur. First, given the evidence that adult neurons are capable of surviving the loss of target neurons for as long as $120 \mathrm{~d}$ (Sofroniew et al., 1990) it may be that trophic interactions generated by the implant are restoring (or even imparting) a functional capacity for circadian rhythm generation to the extra- SCN oscillator that is normally coupled to the SCN. While this explanation does not necessarily depend upon the functional oscillatory capacity within the implant, both the observations that (1) implanted preoptic area tissue, lacking the $\mathrm{SCN}$, is incapable of restoring rhythm- 


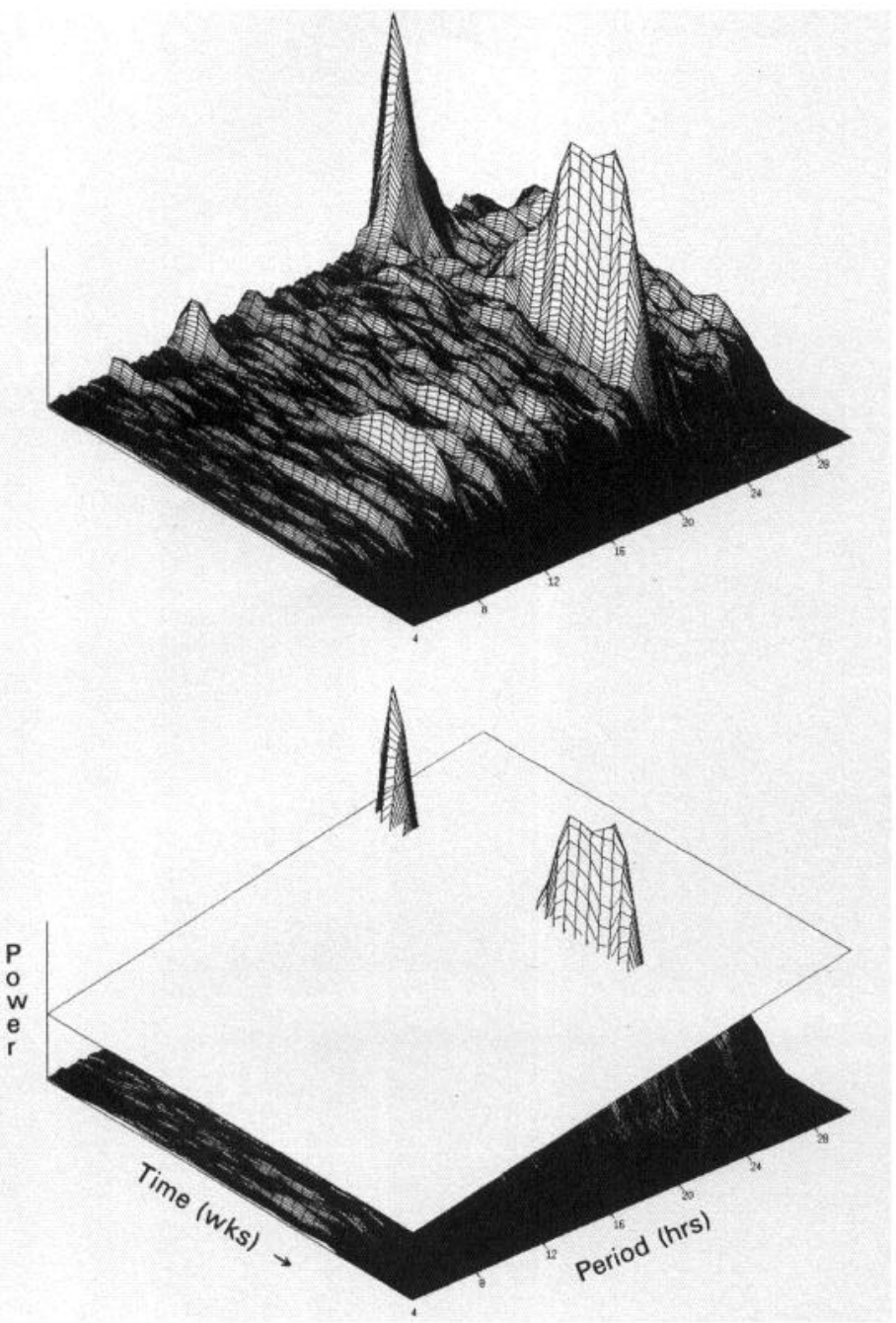

Figure 11. A three-dimensional display of a $\chi^{2}$ periodogram analysis of the wheel-running activity data of the hamster bearing a rat $\mathrm{AH}$ heterograft illustrated in Figure $10 a$ (see Fig. 2 for further detail). Initial circadian rhythmicity $(\tau \approx 24.0 \mathrm{hr}$ ) was disrupted following SCN destruction. A restored circadian rhythm with a mean $\tau$ of $23.66 \mathrm{hr}$ is again evident following heterograft transplantation.

icity and (2) the SCN is a necessary but not sufficient element of functional homografts (Aguilar-Roblero et al., 1988; Boer and Griffioen, 1990) indicate that the posited trophic factors are most likely a specific function of the SCN. However, it would also be assumed that such factor(s) are less efficient in the heterograft preparation than in hamster $\mathrm{AH}$ homografts.

Alternatively, there may be a direct neural recoupling between the $(\mathrm{SCN})$ oscillator within each successful $\mathrm{AH}$ graft and the extra-SCN component in the host. In this case, homografts would be fully effective in recoupling to the extra-SCN oscillator, and, in effect, would replicate the condition of the intact hamster. Heterografts, on the other hand, would couple inadequately or inappropriately, resulting in the expression of the preferred period of the hamster extra-SCN oscillator. Interestingly, the restored periods observed in both heterograft experiments are also commonly observed in hamsters bearing a partially ablated SCN ( $\approx 23.60 \mathrm{hr}$; Pickard and Turek, 1985), and, thus, this period might be common to all "impaired" conditions of the hamster circadian system.

Support for the possibility of a neural recoupling of the im-

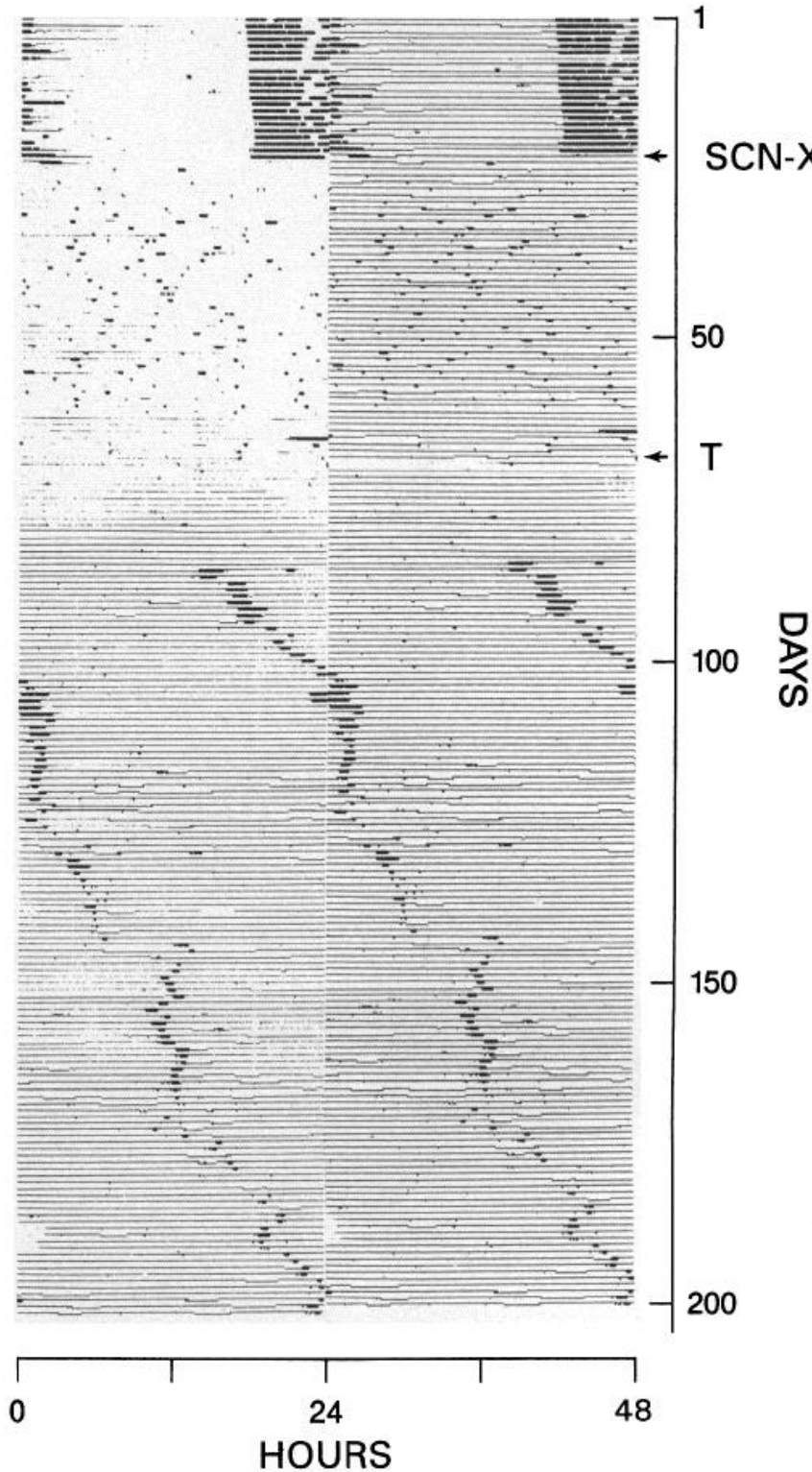

Figure 12. Wheel-running activity record of a hamster bearing a rat $\mathrm{AH}$ heterograft. $S C N-X$ indicates day of SCN lesioning. $T$ indicates day of fetal rat $\mathrm{AH}$ transplantation. The restoration of circadian wheel-running activity is clearly evident following $\mathrm{AH}$ transplantation although the restored period in this animal was exceptional in being rat typical $(\tau=24.24 \mathrm{hr})$.

Table 1. Summary of the $\tau_{D D}$ of intact control animals and $\tau$ of restored circadian rhythm following anterior hypothalamic homografts and heterografts (hamster host in all cases)

\begin{tabular}{lll} 
& $\begin{array}{l}\text { Intact controls } \\
\tau_{\mathrm{DD}}(\mathrm{hr})\end{array}$ & $\begin{array}{l}\text { Fetal AH implant } \\
\tau \text { of restored rhythm }\end{array}$ \\
\hline Hamster & $24.07 \pm 0.01^{a}$ & $24.01 \pm 0.06^{b}$ \\
& $(n=11)$ & $(n=10)$ \\
Mouse & $23.43 \pm 0.02^{\circ}$ & $23.47 \pm 0.08^{d}$ \\
& $(n=23)$ & $(n=9)$ \\
Rat & $24.34 \pm 0.04^{e}$ & $23.60 \pm 0.07^{f}$ \\
& $(n=12)$ & $(n=17)$
\end{tabular}

For details of statistical analysis see text. ${ }^{a} \mathrm{vs}^{b}=$ not significantly different. ${ }^{c}$ vs ${ }^{d}=$ not significantly different. ${ }^{e}$ vs $f=p<0.005 .{ }^{b}$ vs $^{f}=p<0.02 .{ }^{d}$ vs ${ }^{f}$ $=$ not significantly different. ${ }^{b} \mathrm{vs}^{d}=p<0.002$. 

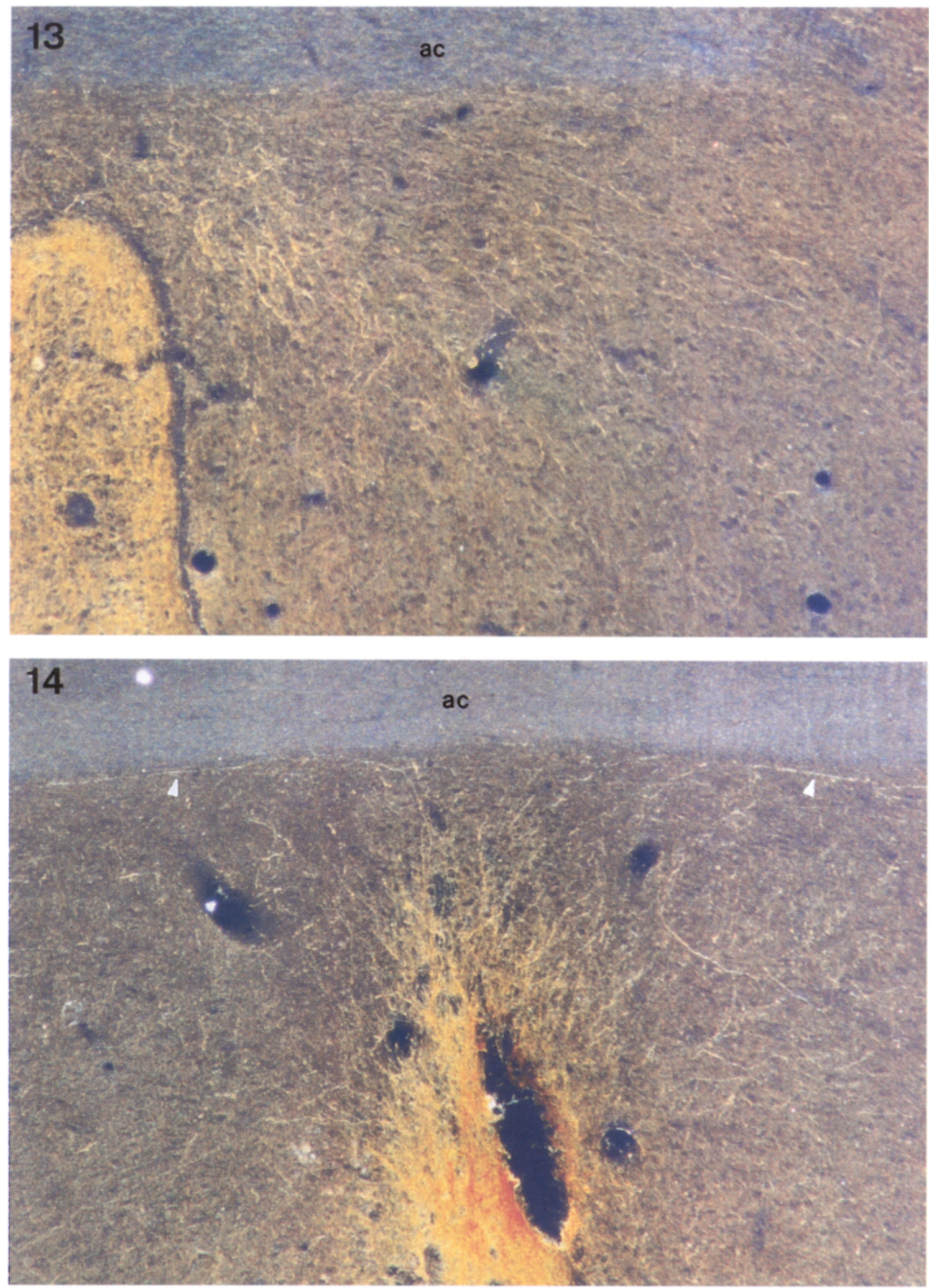

Figure 13. Dark-field photomicrograph illustrating a typical well-vascularized rat AH implant filling the entire third ventricle of the hamster host. The rat implant and many neuronal processes that have grown out from the implant (at a level rostral to this photomicrograph) have been immunocytochemically labeled with the RMO108 monoclonal antibody and are clearly evident in the host hypothalamus. ac, anterior commissure.

Figure 14. Dark-field photomicrograph illustrating neuronal processes labeled immunocytochemically with RMO108, emanating from a rat AH implant situated in the third ventricle of a hamster. Several labeled fibers can be followed leaving the graft dorsally and upon reaching the ventral surface of the anterior commissure $(a c)$ turning laterally to continue along its ventral surface. Many of these fibers extended around the rostral aspect of the anterior commissure and were followed into the bed nucleus of the stria terminalis. 
planted oscillator with a host extra-SCN oscillator may be found in the demonstration of extensive fiber outgrowth from the implant into the host hypothalamus, preoptic area, and septal region. However, the extensive implant fiber outgrowth into the surrounding hypothalamus noted in this $\mathrm{AH}$ heterograft study contrasts with the rather limited neuronal fiber outgrowth reported in AH homograft studies (Lehman et al., 1987; Canbeyli et al., 1991). The difference between these studies is most likely explained by the fact that all heterograft neuronal processes originating from the implant are revealed by the use of speciesspecific antibodies. In the homograft studies, only a fraction of the implant is labeled when antibodies to SCN peptides are used for tracing or when anterograde neuronal tracers are placed on or injected into the graft (Sollars and Pickard, 1994). Nevertheless, the possibility that fiber outgrowth from neural heterografts might be generally more extensive than fiber outgrowth from neural homografts cannot as yet be completely eliminated.

Although the idea that $\tau$ is not solely a function of the SCN is consistent with the data obtained in the current series of experiments, it stands in apparent contrast to the hamster allograft study by Ralph et al. (1990), in which transplantations using the $\tau$ mutant hamster demonstrated that the restored period was singularly determined by the genotype of the donor. The authors interpreted this result as an indication "that either the SCN is essentially autonomous in determining the primary characteristics of rhythmicity in hamsters or that the host brain fails to make the connections with the tissue graft that are required for the brain to influence this period" (Ralph et al., 1990). However, while their result clearly demonstrates that the SCN plays an active role in the generation of circadian rhythmicity, and unequivocally demonstrates that the SCN is the only component of the hamster's circadian system that has been altered by the tau mutation, it leaves unaddressed the possibility that an extraSCN oscillator is a fundamental component in the determination of the species-typical $\tau$ of the hamster.

The counterargument procecds as follows. If, for cxample, the extra-SCN component of the hamster circadian pacemaker generates a period of approximately $23.5 \mathrm{hr}$ (to be consistent with the suggestion raised by the current heterograft results), the role of the hamster SCN may be a fine-tuning of that period en route to its overt expression. Accordingly, a normal hamster SCN tunes it slightly upward to $24 \mathrm{hr}$, whereas a heterozygote tau mutated SCN modifies it downward to $22 \mathrm{hr}$ and a homozygote tau mutated SCN drives it further down to $20 \mathrm{hr}$. Clearly, if the tau mutation has altered only the circadian component resident in the SCN (as shown in allograft experiments), then the genotype of the SCN-ablated host becomes irrelevant. Both the normal SCNx hamster and the tau mutant SCNx hamster retain an unmutated extra-SCN oscillator with a period (in this example) of $23.5 \mathrm{hr}$, and are indistinguishable from one another as hosts in the allograft transplantation experiments. Thus, analogous to the cockroach optic lobe transplantation experiment (Page, 1982), apparent determination of the period by the implant is necessary, but does not provide sufficient evidence for an autonomous or even hierarchically predominant pacemaker within the transplanted locus. However, the significant modification of the period in the homozygous $\tau$ mutant SCN may provide a valuable tool for further examination of the coupling between this oscillator and others in the hamster, whether those extraSCN oscillators are subordinate or commensurate.

In summary, the current study has firmly established the capacity of $\mathrm{AH}$ heterografts to restore circadian rhythmicity to
SCN-lesioned hosts, and has provided an evaluation of the donor fiber outgrowth into the host hypothalamus, which has so far been restricted in the $\mathrm{AH}$ homograft and allograft preparations. The inability to demonstrate fiber outgrowth in homografts has prompted the suggestion by some that the grafts communicate with the host via a diffusible substance (Lehman et al., 1987). The extent of fiber outgrowth noted in the present study is sufficiently robust and sustained to strengthen the possibility that the observed restoration of locomotor rhythmicity is a consequence of neuronal donor/host integration, consistent with the time course of graft fiber outgrowth, which precedes the time course of behavioral restoration (Sollars and Pickard, 1993). Although the observed graft/host integration favors a synaptic mode underlying the restored behavior, it does not eliminate the possibility of humoral communication.

Finally, the two general (albeit diametrically opposed) interpretations of the behavioral data provide models for which a number of experimental tests can be devised. A critical determinant will be the capacity of the hamster $\mathrm{AH}$ graft to generate a hamster-typical period in a heterograft preparation. Additionally, a more systematic analysis of rat $\mathrm{AH}$ implants, comparing grafts that restore periods of $<24 \mathrm{hr}$ to those that successfully restore a more rat-typical period of $24.3 \mathrm{hr}$, may provide insight into the possibility that the rat circadian system has a different hierarchical organization than that of the hamster or mouse. A clarification of this issue will have especially broad ramifications, considering the variety of anatomical differences already apparent between the hamster and rat suprachiasmatic nuclei and retinohypothalamic projections (Pickard and Silverman, 1981; Card and Moore, 1984; van den Pol and Tsujimoto, 1985; Levine et al., 1991) and the burgeoning generation of mouse models of circadian rhythmicity as an avenue to the molecular analysis of mammalian circadian rhythmicity (Pickard et al., 1994; Takahashi et al., 1994; Vitaterna et al., 1994). Thus, the combined $\mathrm{AH}$ heterograft results may be most valuable in pointing the way for further investigations to determine the autonomous function of the suprachiasmatic nucleus and the complete locus of the mammalian biological clock.

\section{References}

Aguilar-Roblero R, Morin LP, Moore RY (1988) Is the fetal SCN sufficient to induce rhythm recovery when transplanted to $\mathrm{SCN}$-lesioned hamsters? Soc Neurosci Abstr 14:49.

Balaban E, Teillet M-A, Le Douarin N (1988) Application of the quailchick chimera system to the study of brain development and behavior. Science 241:1339-1342.

Boer GJ, Griffioen HA (1990) Developmental and functional aspects of grafting of the suprachiasmatic nucleus in the Brattleboro rat. Eur J Morphol 28:330-345.

Canbeyli RS, Lehman M, Silver R (1991) Tracing SCN graft efferents with DiI. Brain Res 554:15-19.

Card JP, Moore RY (1984) The suprachiasmatic nucleus of the golden hamster: immunocytochemical analysis of cell and fiber distribution. Neuroscience 13:415-431.

Cohen RA, Albers HE (1991) Disruption of human circadian and cognitive function following a discrete hypothalamic lesion. Neurology 41:726-728.

Dark J, Pickard GE, Zucker I (1985) Persistence of circannual rhythms in ground squirrels with lesions of the suprachiasmatic nuclei. Brain Res 332:201-207.

DeCoursey PJ, Buggy J (1988) Restoration of circadian locomotor activity in arrhythmic hamsters by fetal SCN transplants. Comp Endocrinol 7:49-54.

Gerkema MP, Groos GA, Daan S (1990) Differential elimination of circadian and ultradian rhythmicity by hypothalamic lesions in the common vole, Microtus arvalis. I Biol Rhythms 5:81-95.

Griffioen HA, Duindam H, van der Woude TP, Rietveld WJ, Boer GJ 
(1993) Functional development of fetal suprachiasmatic nucleus grafts in suprachiasmatic nucleus-lesioned rats. Brain Res Bull 31: $145-160$.

Kawamura H, Nihonmatsu I (1985) The suprachiasmatic nucleus as a circadian rhythm generator. In: Circadian clocks and zeitgebers (Hiroshige T, Honma K, eds), pp 55-63. Sapporo: Hokkaido UP.

Klassen H, Lund RD (1988) Anatomical and behavioral correlates of a xenograft-mediated pupillary reflex. Exp Neurol 102:102-108.

Lee VM-Y, Carden MJ, Schlaepfer WW, Trojanowski JQ (1987) Monoclonal antibodies distinguish several differentially phosphorylated states of the two largest rat neurofilament subunits (NF-H and NF$\mathrm{M})$ and demonstrate their existence in the normal nervous system of adult rats. J Neurosci 7:3474-3488.

Lehman MN, Silver R, Gladstone WR, Kahn RM, Gibson M, Bittman EL (1987) Circadian rhythmicity restored by neural transplant. Immunocytochemical characterization of the graft and its integration with the host brain. J Neurosci 7:1626-1638.

Levine JD, Weiss ML, Rosenwasser AM, Miselis RR (1991) Retinohypothalamic tract in the female albino rat: a study using horseradish peroxidase conjugated to cholera toxin. J Comp Neurol 306:344-360.

I und RD, Chang F-LF, Hankin MH, Lagenaur CF (1985) Use of a species-specific antibody for demonstrating mouse neurons transplanted to rat brains. Neurosci Lett 61:221-226.

Meijer JH, Rietveld WJ (1989) Neurophysiology of the suprachiasmatic circadian pacemaker in rodents. Physiol Rev 69:671-707.

Moore RY, Klein DC (1974) Visual pathways and the central neural control of a circadian rhythm in pineal N-acetyltransferase activily. Brain Res 71:17-33.

Page TL (1978) Interactions between bilaterally paired components of the cockroach circadian system. J Comp Physiol 124:225-236.

Page TL (1981) Effects of localized low-temperature pulses on the cockroach circadian pacemaker. Am J Physiol 240:R144-R150.

Page TL (1982) Transplantation of the cockroach circadian pacemaker. Science 216:73-76.

Pickard GE, Silverman AJ (1981) Direct retinal projections to the hypothalamus, piriform cortex and accessory optic nuclei in the golden hamster as demonstrated by a sensitive anterograde horseradish peroxidase technique. J Comp Neurol 196:155-172.

Pickard GE, Turek FW (1985) Effects of partial destruction of the suprachiasmatic nuclei on two circadian parameters: wheel-running activity and short-day induced testicular regression. J Comp Physiol 156:803-815.

Pickard GE, Ralph MR, Menaker M (1987) The intergeniculate leaflet partially mediates effects of light on circadian rhythms. J Biol Rhythms 2:35-56.

Pickard GF, Sollars PI, Rinchik EM, Nolan P, Bucan M (1994) Mutagenesis screen identifies a mouse (187) with altered circadian rhythms. Soc Res Biol Rhythms 4:58.

Pittendrigh CS, Daan S (1976) $\Lambda$ functional analysis of circadian pacemakers in nocturnal rodents. I. The stability and lability of spontaneous frequency. J Comp Physiol 106:223-252.

Ralph MR, Foster RG, Davis FC, Menaker M (1990) Transplanted suprachiasmatic nucleus determines circadian period. Science 247 : 975-978.

Reppert SM, Schwartz WJ (1984) The suprachiasmatic nuclei of the fetal rat: characterization of a functional circadian clock using ${ }^{14} \mathrm{C}$ labeled deoxyglucose. J Neurosci 4:1677-1682.

Reppert SM, Perlow MJ, Ungerleider LG, Mishkin M, Tamarkin L,
Orloff DG, Hoffman HJ, Klein DC (1981) Effects of damage to the suprachiasmatic area of the anterior hypothalamus on the daily melatonin and cortisol rhythms in the rhesus monkey. $\mathbf{J}$ Neurosci $1: 1414-1425$.

Roberts MH, Bernstein MF, Moore RY (1987) Differentiation of the suprachiasmatic nucleus in fetal rat anterior hypothalamic transplants in oculo. Dev Brain Res 32:59-66.

Rosenwasser AM (1988) Behavioral neurobiology of circadian pacemakers: a comparative perspective. Prog Psychobiol Physiol Psychol 13:155-226.

Rusak B (1977) The role of the suprachiasmatic nuclei in the generation of circadian rhythms in the golden hamster, Mesocricetus auratus. J Comp Physiol 118:145-164.

Saitoh Y, Nihonmatsu I, Kawamura H (1990) Location of the suprachiasmatic nucleus grafts in rats which restored circadian rhythmicity after transplantation. Neurosci Lett 118:45-48.

Saitoh Y, Matsui Y, Nihonmatsu I, Kawamura H (1991) Cross-species transplantation of the suprachiasmatic nuclei from rats to siberian chipmunks (Eutamias sibiricus) with suprachiasmatic lesions. Neurosci Lett 123:77-81.

Sawaki Y, Nihonmatsu I, Kawamura H (1984) Transplantation of the neonatal suprachiasmatic nuclei into rats with complete bilateral suprachiasmatic lesions. Neurosci Res 1:67-72.

Schwartz WJ, Zimmerman P (1991) Lesions of the suprachiasmatic nucleus disrupt circadian locomotor rhythms in the mouse. Physiol Behav 49:1283-1287.

Sofroniew MV, Galletly NP, Isacson O, Svendsen CN (1990) Survival of adult basal forebrain cholinergic neurons after loss of target neurons. Science 247:338-342.

Sokolove PG, Bushell WN (1978) The chi square periodogram: its utility for analysis of circadian rhythms. J Theor Biol 72:131-160.

Sollars PJ, Pickard GE (1993) Time course of fiber outgrowth from fetal anterior hypothalamic heterografts. Brain Res 614:212-219.

Sollars PJ, Pickard GE (1994) Neural heterografts as a model for the study of mammalian circadian behavior. In: Neural transplantation, CNS neuronal injury and regeneration: recent advances (Marwah J, Teitelbaum P, Prasad KN, eds), pp 161-181. Boca Raton, FL: CRC.

Stephan FK, Zucker I (1972) Circadian rhythms in drinking behavior and locomotor activity of rats are eliminated by hypothalamic lesions. Proc Natl Acad Sci USA 69:1583-1586.

Takahashi JS, Pinto LH, Vitaterna MH (1994) Forward and reverse genetic approaches to behavior in the mouse. Science 264:17241733.

Turek FW (1985) Circadian neural rhythms in mammals. Annu Rev Physiol 47:49-64.

van den Pol AN, Tsujimoto KL (1985) Neurotransmitters of the hypothalamic suprachiasmatic nucleus: immunocytochemical analysis of 25 neuronal antigens. Neuroscience 15:1049-1086.

Vitaterna MH, King DP, Chang A-M, Kornhauser JM, Lowrey PL, McDonald JD, Dove WF, Pinto LH, Turek FW, Takahashi JS (1994) Mutagenesis and mapping of a mouse gene, clock, essential for circadian behavior. Science 264:719-725.

Wictorin K, Lagenaur CF, Lund RD, Bjorklund A (1991) Efferent projections to the host brain from intrastriatal striatal mouse-to-rat grafts: time course and tissue-type specificity as revealed by a mouse specific neuronal marker. Eur J Neurosci 3:86-101.

Zimmerman NH, Menaker M (1979) The pineal gland: a pacemaker within the circadian system of the house sparrow. Proc Natl Acad Sci USA 76:999-1003. 